\title{
Prévalence réelle de la brucellose bovine dans le centre de la Côte d'Ivoire
}

\author{
M. Sanogo ${ }^{1}$ B. Cissé ${ }^{1}$ M. Ouattara ${ }^{1}$ K. Walravens ${ }^{2}$ \\ N. Praet ${ }^{3}$ D. Berkvens ${ }^{3}$ E. Thys ${ }^{3 *}$
}

\section{Mots-clés}

Bovin - Brucella - Brucellose Technique immunologique Morbidité - Côte d'Ivoire.

\begin{abstract}
Résumé
Lors de l'enquête de sérosurveillance active de la peste bovine organisée en Côte d'Ivoire de juillet à août 2005, 660 sérums ont été collectés dans les régions administratives du N'Zi Comoé et des Lacs, au centre du pays. Ces sérums ont été utilisés pour faire une estimation de la prévalence réelle de la brucellose bovine en utilisant une approche statistique bayésienne avec quatre tests de diagnostic sérologiques : la séro-agglutination lente de Wright (SAW), le test du rose bengale (TRB), l'Elisa indirect (iElisa) et le test de fixation du complément (TFC). Les résultats de ce dernier n'ont pas été pris en compte dans cette étude. Les analyses sérologiques ont donné des séroprévalences individuelles de 6,2 p. 100 à la SAW, 5,1 p. 100 au TRB et 7,0 p. 100 à I'iElisa. L'analyse bayésienne faite avec ces mêmes résultats sérologiques a permis d'obtenir une estimation de la prévalence réelle de la brucellose de 8,8 p. 100 (intervalle de crédibilité à 95 p. $100: 5,0-16,4)$. Ces résultats constituent une bonne indication de l'importance de la brucellose dans cette partie de la Côte d'Ivoire et confirment l'attention qui doit être accordée à cette maladie.
\end{abstract}

\section{INTRODUCTION}

L'élevage occupe une place importante pour les populations africaines. Le développement de cet élevage est cependant sous la contrainte de nombreux facteurs dont les contraintes pathologiques. Outre leur impact sur la santé des animaux, certaines de ces pathologies, peuvent aussi causer des problèmes de santé publique. C'est le cas de la brucellose $(1,6,10)$, maladie fortement pathogène, due à des bactéries du genre Brucella. Affectant aussi bien les humains que de nombreuses espèces animales domestiques et sauvages, elle est considérée comme l'une des zoonoses les plus répandues dans le monde $(8,17)$. Chez les animaux, la brucellose

1. Laboratoire national d'appui au développement agricole (Lanada), Abidjan, Côte d'Ivoire.

2. Département de bactériologie et d'immunologie, Centre d'étude et de recherches vétérinaires et agrochimiques (Cerva), Bruxelles, Belgique.

3. Institut de médecine tropicale, département de santé animale, Anvers, Belgique.

* Auteur pour la correspondance

Institut de médecine tropicale, département de santé animale, Nationalestraat 155, Anvers, Belgique.

Tél. : +32 32476392 ; fax : +3232476268

E-mail : ethys@itg.be bovine, essentiellement due à Brucella abortus, reste la plus répandue en Afrique $(7,15)$. Connue depuis de nombreuses années en Côte d'Ivoire, cette maladie du bétail y est considérée comme une dominante pathologique (4). A ce titre, diverses études épidémiologiques lui ont ainsi été consacrées, mais elles sont relativement peu nombreuses et peu récentes $(4,6,13,18)$. S'inscrivant dans la continuité de l'étude de Thys et coll. en 2005 (24), la présente étude a eu pour objectif de contribuer à l'actualisation des connaissances épidémiologiques sur la brucellose bovine dans le centre de la Côte d'Ivoire et, notamment, dans les régions du N'Zi Comoé et des Lacs.

\section{MATERIEL ET METHODES}

\section{Contexte de l'étude}

Les régions du N'Zi Comoé et des Lacs sont localisées au centre du pays, à environ $300 \mathrm{~km}$ au nord d'Abidjan, la capitale économique du pays. Elles sont subdivisées administrativement en plusieurs départements (figure 1). Le centre de la Côte d'Ivoire est une zone intermédiaire entre le nord (relativement sec) et le sud (humide) du pays, aussi bien au niveau du climat que de la végétation. 


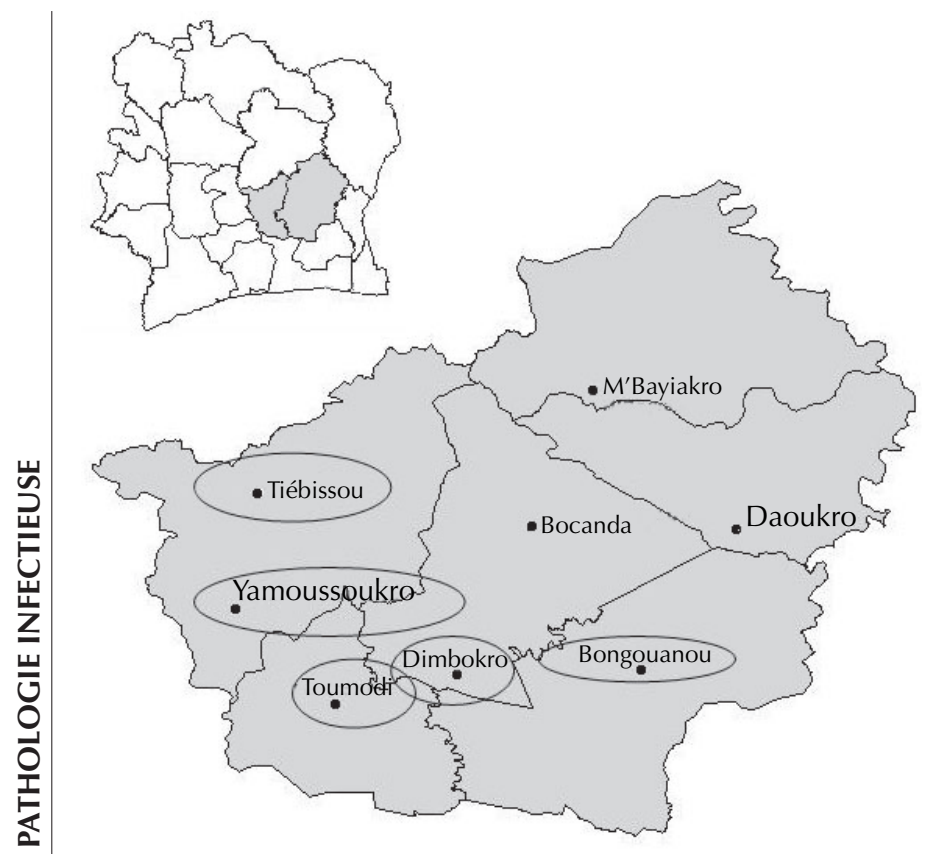

Figure 1 : zone d'étude avec les localités de Bongouanou, Dimbokro, Toumodi, Yamoussoukro et Tiébissou (Côte d'Ivoire).

Habituellement, cette zone est occupée par des élevages bovins de type sédentaire avec parfois quelques troupeaux transhumants. Le mode d'élevage dominant est de type traditionnel sédentaire avec le recours au pâturage comme principale source alimentaire. Le cheptel est constitué de taurins (Bos taurus) (N'Dama, Baoulé) et de zébus (Bos indicus) transhumants. On y trouve aussi des métis issus de croisements entre zébus et taurins.

\section{Données de l'étude}

Les données utilisées dans cette étude sont issues du N'Zi Comoé et des Lacs. Elles ont été collectées lors d'une enquête de sérosurveillance organisée en 2005 dans le cadre des activités du programme panafricain de contrôle des épizooties en Côte d'Ivoire. Cette enquête a eu pour objectif de détecter la présence de la peste bovine, avec un niveau de confiance de 95 p. 100 en supposant une prévalence de 1 p. 100 au niveau des troupeaux et de 5 p. 100 au sein des troupeaux du pays (14). Un échantillon aléatoire de 15 animaux de plus d'un an d'âge a été sélectionné dans chaque troupeau sélectionné. A chaque fois, des informations complémentaires (race, sexe, âge, localité, nom du propriétaire) ont été collectées.

Ainsi, 660 sérums bovins ont été collectés dans les 44 troupeaux localisés dans les cinq départements de ces deux régions, puis utilisés dans le cadre de cette étude de prévalence (tableau I). Cet échantillon était composé essentiellement de femelles issues de troupeaux traditionnels sédentaires, avec environ deux tiers des bovins métis et zébus, d'une part, et deux tiers des bovins de plus de trois ans, d'autre part (tableau II).

\section{Analyses sérologiques}

Les sérums ont été analysés au Centre d'étude et de recherches vétérinaires et agrochimiques (Cerva, Bruxelles, Belgique) en utilisant quatre tests sérologiques de diagnostic de la brucellose : la séro-agglutination lente de Wright (SAW), le test au rose bengale (TRB), le test d'Elisa indirect (iElisa) et le test de fixation du complément (TFC). Ces tests ont été appliqués selon Shey-Njila et coll. (22), et Thys et coll. (24).

\section{Tableau I}

Nombre de troupeaux et de sérums collectés par région et par département

\begin{tabular}{llcc} 
Région & Département & $\begin{array}{c}\text { Nb. de } \\
\text { troupeaux }\end{array}$ & $\begin{array}{c}\text { Nb. de sérums } \\
\text { collectés }\end{array}$ \\
\hline $\mathrm{N}^{\prime} \mathrm{Zi}$ & Bongouanou & 15 & 225 \\
Comoé & Dimbokro & 12 & 180 \\
Lacs & Tiébissou & 7 & 105 \\
& Toumodi & 5 & 75 \\
& Yamoussoukro & 5 & 75 \\
\hline \multirow{2}{*}{ Total } & & 44 & 660
\end{tabular}

\section{Tableau II}

Composition de l'échantillon par sexe, âge et race

\begin{tabular}{|c|c|c|c|c|c|}
\hline \multirow[t]{2}{*}{ Sexe } & \multirow[t]{2}{*}{ Age } & \multicolumn{3}{|c|}{ Race } & \multirow[t]{2}{*}{ Total } \\
\hline & & Taurin & Zébu & Zébu x Taurin & \\
\hline \multirow[t]{2}{*}{ Femelle } & 1 à 3 ans & 67 & 20 & 127 & 214 \\
\hline & $>3$ ans & 115 & 20 & 162 & 297 \\
\hline Total femelle & & 182 & 40 & 289 & 511 \\
\hline \multirow[t]{2}{*}{ Mâle } & 1 à 3 ans & 42 & 14 & 72 & 128 \\
\hline & $>3$ ans & 4 & 4 & 13 & 21 \\
\hline Total mâle & & 46 & 18 & 85 & 149 \\
\hline $\begin{array}{l}\text { Total (femelle } \\
+ \text { mâle) }\end{array}$ & & 228 & 58 & 374 & 660 \\
\hline
\end{tabular}

\section{Analyse statistique bayésienne}

Pour l'estimation de la prévalence réelle, une approche bayésienne a été utilisée $(5,19)$. Cette technique d'analyse statistique a consisté à combiner les résultats des tests sérologiques dans un modèle dit bayésien avec des informations d'experts (information a priori) sur la sensibilité et la spécificité des tests utilisés, pour obtenir une estimation de la prévalence réelle a posteriori. Cette analyse a été faite à l'aide d'un modèle mathématique multinomial construit à partir des différentes combinaisons possibles de résultats avec les différents tests sérologiques pour chaque sérum. Ce modèle a été basé sur les probabilités conditionnelles (par exemple, la probabilité qu'un individu teste positif au test 2, étant donné qu'il était infecté et positif au test 1) et les éventuelles dépendances conditionnelles entre les tests appliqués dans l'analyse (5). Les informations a priori, utiles pour permettre d'avoir des estimations, ont été traduites sous la forme de contraintes sur les valeurs possibles des caractéristiques (sensibilité et spécificité) des tests employés dans ce modèle multinomial (annexe). La concordance de celles-ci avec les résultats d'analyse sérologique ainsi que la validation du modèle utilisé ont été évaluées parallèlement au cours de l'analyse. Les opinions d'expert utilisées dans l'analyse ont été les mêmes que celles utilisées par Thys et coll. (24) et extraites de la synthèse faite par Nielsen sur le diagnostic sérologique de la brucellose (16) (tableau III). Cette analyse a été faite à l'aide du logiciel Winbugs $®(5,19,23)$. En raison d'un grand 
nombre de résultats ininterprétables au TFC, les résultats de ce dernier test n'ont pas été inclus dans l'analyse.

\section{Tableau III}

Informations a priori utilisées pour l'analyse bayésienne

\begin{tabular}{lrc} 
Test & Sensibilité $(\%)$ & Spécificité $(\%)$ \\
\hline SAW & $29,1-100$ & $99,2-100$ \\
TRB & $21-98,3$ & $68,8-100$ \\
TFC & $23-97,1$ & $30,6-100$ \\
iElisa & $92,5-100$ & $90,6-100$
\end{tabular}

Source : Nielsen, 2002

SAW : séro-agglutination lente de Wright ; TRB : test du rose bengale ; TFC : test de fixation du complément; iElisa : Elisa indirect

\section{Annexe}

Modèle bayésien utilisé pour l'estimation de la prévalence réelle avec les informations a priori de Nielsen sur la sensibilité et la spécificité du test de séro-agglutination lente de Wright.

Les p[i] sont les différents résultats possibles en fonction du nombre de tests utilisés.

Les $\theta[i]$ sont les probabilités conditionnelles.

model

\{

res[1:8] dmulti(p[1:8],n)

$\mathrm{p}[1]<-\theta[1] *(1-\theta[2])^{*}(1-\theta[5])^{*}(1-\theta[11])+(1-\theta[1])^{*} \theta[3]^{*} \theta[6] * \theta[12]$

$\mathrm{p}[2]<-\theta[1] *(1-\theta[2])^{*}(1-\theta[5])^{*} \theta[11]+(1-\theta[1])^{*} \theta[3]^{*} \theta[6]^{*}(1-\theta[12])$

$\mathrm{p}[3]<-\theta[1] *(1-\theta[2])^{*} \theta[5] *(1-\theta[10])+(1-\theta[1]) * \theta[3]^{*}(1-\theta[6])^{*} \theta[13]$

$\mathrm{p}[4]<-\theta[1] *(1-\theta[2])^{*} \theta[5]^{*} \theta[10]+(1-\theta[1])^{*} \theta[3]^{*}(1-\theta[6])^{*}(1-\theta[13])$

$\mathrm{p}[5]<-\theta[1] * \theta[2] *(1-\theta[4]) *(1-\theta[9])+(1-\theta[1]) *(1-\theta[3]) * \theta[7] * \theta[14]$

$\mathrm{p}[6]<-\theta[1] * \theta[2] *(1-\theta[4]) * \theta[9]+(1-\theta[1]) *(1-\theta[3]) * \theta[7] *(1-\theta[14])$

$\mathrm{p}[7]<-\theta[1]^{*} \theta[2] *{ }^{*} \theta[4] *(1-\theta[8])+(1-\theta[1])^{*}(1-\theta[3])^{*}(1-\theta[7])^{*} \theta[15]$

$\mathrm{p}[8]<-\theta[1] * \theta[2]^{*} \theta[4] * \theta[8]+(1-\theta[1]) *(1-\theta[3]) *(1-\theta[7]) *(1-\theta[15])$

res1 [1:8] dmulti(p[1:8],n)

for (i in $1: 8)$

di $[i]<-\operatorname{res}[i] * \log (\max (\operatorname{res}[i], 1) /(p[i] * n))$

di2 [i]<-res1 [i]* $\log (\max (\operatorname{res} 1[i], 1) /(p[i] * n))$

$\mathrm{G} 0<-2 * \operatorname{sum}(\operatorname{di}[])$

$\mathrm{Gt}<-2 * \operatorname{sum}(\mathrm{di} 2[])$

bayesp $<-\operatorname{step}(\mathrm{G} 0-\mathrm{G} t)$

$\theta[1] \sim \operatorname{dbeta}(1,1)$

$\theta[2] \sim \operatorname{dbeta}(1,1) \mathrm{I}(0.291,1)$

$\theta[3] \sim \operatorname{dbeta}(1,1) \mathrm{l}(0.992,1)$

$\theta[4] \sim \operatorname{dbeta}(1,1)$

$\theta[5] \sim \operatorname{dbeta}(1,1)$

$\theta[6] \sim \operatorname{dbeta}(1,1)$

$\theta[7] \sim \operatorname{dbeta}(1,1$

$\theta[8] \sim \operatorname{dbeta}(1,1)$

$\theta[9] \sim \operatorname{dbeta}(1,1)$

$\theta[10] \sim \operatorname{dbeta}(1,1)$

$\theta[11] \sim \operatorname{dbeta}(1,1)$

$\theta[12] \sim \operatorname{dbeta}(1,1)$

$\theta[13] \sim \operatorname{dbeta}(1,1)$

$\theta[14] \sim \operatorname{dbeta}(1,1)$

$\theta[15] \sim \operatorname{dbeta}(1,1)$

$\mathrm{se}[1]<-\theta[2]$

sp $[1]<-\theta[3]$

se[2]<- $\theta[2]^{*} \theta[4]+(1-\theta[2])^{*} \theta[5]$

sp $[2]<-\theta[3]^{*} \theta[6]+(1-\theta[3]) * \theta[7]$

se $[3]<-\theta[2] * \theta[4] * \theta[8]+\theta[2] *(1-\theta[4]) * \theta[9]+(1-\theta[2]) * \theta[5] * \theta[10]+(1-$ $\theta[2])^{*}(1-\theta[5])^{*} \theta[11]$

$\operatorname{sp}[3]<-\theta[3] * \theta[6] * \theta[12]+\theta[3] *(1-\theta[6]) * \theta[13]+(1-\theta[3]) * \theta[7] * \theta[14]+(1-$ $\theta[3])^{*}(1-\theta[7]) * \theta[15]$

list $($ res $=c(542,5,18,8,18,3,2,15), n=611)$
RESULTATS

\section{Résultats sérologiques}

Les résultats sérologiques bruts obtenus sont résumés dans le tableau IV. Les séroprévalences par test sont présentées dans le tableau V.

\section{Résultats de l'analyse statistique bayésienne}

L'analyse bayésienne à partir des résultats sérologiques a permis d'obtenir une estimation de la prévalence réelle avec un intervalle de crédibilité de 95 p. 100. Les meilleures estimations ont été obtenues en utilisant les informations a priori fournies par Nielsen sur la SAW. Les résultats sont résumés dans le tableau VI.

\section{Tableau IV}

Résultats sérologiques bruts pour les différents tests inclus dans l'analyse

\begin{tabular}{cccc} 
SAW & TRB & iElisa & Nb. de sérums \\
\hline 0 & 0 & 0 & 542 \\
0 & 0 & 1 & 18 \\
0 & 1 & 0 & 5 \\
0 & 1 & 1 & 8 \\
1 & 0 & 0 & 18 \\
1 & 0 & 1 & 2 \\
1 & 1 & 0 & 3 \\
1 & 1 & 1 & 15 \\
& & & 611
\end{tabular}

SAW : séro-agglutination lente de Wright ; TRB : test du rose bengale ; iElisa : Elisa indirect

0 : test négatif $; 1:$ test positif

\section{Tableau V}

Séroprévalences et intervalles de crédibilité (IC) à 95\%

\begin{tabular}{lccc} 
Test & Nb. de positifs & Séroprévalence (\%) & IC \\
\hline SAW & 38 & 6,2 & $4,4-8,4$ \\
TRB & 31 & 5,1 & $3,5-7,1$ \\
iElisa & 43 & 7,0 & $5,1-9,4$
\end{tabular}

SAW : séro-agglutination lente de Wright ; TRB : test du rose bengale ; iElisa : Elisa indirect

\section{Tableau VI}

Prévalence réelle et intervalle de crédibilité (IC) à 95\%

Test appliqué Prévalence réelle (\%) IC

SAW

TRB

$5,0-16,4$

iElisa

SAW : séro-agglutination lente de Wright ; TRB : test du rose bengale ; iElisa : Elisa indirect 


\section{DISCUSSION}

Les estimations de séroprévalence se sont appuyées sur des données collectées dans un autre cadre et avec un objectif différent de l'estimation d'une prévalence. L'utilisation de telles données a permis d'obtenir de façon rapide et à moindre coût des informations sur la brucellose bovine. Cependant, elle peut présenter quelques limites (20). La taille de l'échantillon ainsi que la bonne distribution spatiale des troupeaux sélectionnés ont paru satisfaisant pour utiliser ces données. Par ailleurs, ce type de données a été déjà utilisé dans des études de prévalences (25).

En considérant les différents tests individuellement, la séroprévalence de la brucellose dans la zone d'étude se situait entre 3,5 à 9,4 p. 100, avec respectivement des estimations de 6,2, 5,1 et 7,0 p. 100 pour les tests SAW, TRB et iElisa. Cela confirme la présence de la brucellose dans cette zone. Cependant dans le cas de la brucellose, l'application isolée des tests sérologiques usuels tel que la SAW, le TRB et l'iElisa ne donnent pas d'information suffisante sur le statut réel des animaux et rend difficile l'estimation de la prévalence. Par ailleurs, bien que la vaccination susceptible d'inférer avec ces tests ne soit pas pratiquée en Côte d'Ivoire, d'autres organismes ayant des caractéristiques antigéniques proches, comme Yersinia enterocolitica 0:9, Xanthomonas maltophilia et Salmonella urbana, sont capables de donner une sérologie faussement positive (21).

En l'absence de tests parfaits pour le diagnostic de la brucellose, une combinaison de tests sérologiques a été utilisée afin d'obtenir des estimations plus fiables, de réduire les erreurs de classification et d'améliorer la sensibilité et la spécificité du diagnostic. Une prévalence réelle de 8,8 p. 100 (intervalle de crédibilité de 95 p. 100 : $5,0-16,4)$ a été ainsi obtenue. Cette estimation était supérieure à celles fournies par les tests individuels. Elle était supérieure, voire le double, de celle obtenue par Thys et coll. en 2005 (24) dans le sud de la Côte d'Ivoire (région des Lagunes) en utilisant cette même approche.

Comparativement au sud, la partie centrale de la Côte d'Ivoire est plus favorable à l'élevage des bovins. De plus grands espaces et pâturages favorisent des densités et concentrations de troupeaux plus grandes ainsi que les mouvements d'animaux, facteurs pouvant augmenter le risque de contact et de dissémination de l'infection (1). Par ailleurs, avec la crise sociopolitique que vit la Côte d'Ivoire depuis 2002, les régions du N'Zi Comoé et des Lacs ont accueilli d'autres troupeaux venus du Nord pour se sédentariser dans ces zones, ce qui a pu influencer également la prévalence estimée par la présente étude. Près de deux tiers des animaux inclus dans cette étude étaient âgés d'au moins trois ans, ce qui pouvait avoir une influence ; la prévalence de la brucellose serait plus élevée chez les animaux âgés $(2,12)$. Cette estimation, bien que proche des valeurs de 11 à 14 p. 100 et de 10,9 p. 100 obtenues respectivement par Angba et coll. en 1987 (4) et Pilo-Moron et coll. en 1979 (18) en Côte d'Ivoire, reste difficilement comparable à celles-ci en raison de la différence d'approche. Ces estimations étaient basées sur un seul test ou tout au plus sur deux tests en complémentarité.

D'autres études de prévalences ont été également faites sur la brucellose bovine en Afrique et dans la sous-région ouest-africaine et ont permis d'obtenir diverses estimations $(2,3,10,15$, 25). La différence des tests appliqués, les différentes échelles de ces études et surtout la différence de méthodologie rendent difficile toute comparaison avec l'estimation de la présente étude. Dans la plupart des cas précédents, un seul test (TRB ou TFC ou Elisa ou SAW) ou parfois une association de tests a été utilisé. Ainsi, dans des études récentes, Delafosse et coll. (9), et
Faye et coll. (12) ont respectivement estimé des prévalences de 2,6 p. 100 au Tchad et de 15,8 p. 100 en Ouganda en s'appuyant sur des résultats du TRB ou du TRB associé au TFC. Shey-Njila et coll. (22) ont également trouvé une prévalence de l'ordre de 10 p. 100 en s'appuyant sur des résultats d'Elisa au Cameroun.

\section{CONCLUSION}

Cette étude a eu pour objectif d'apporter de nouvelles connaissances sur l'épidémiologie de la brucellose bovine en Côte d'Ivoire. Elle a permis de confirmer la présence de cette maladie dans la zone Centre du pays, avec une prévalence réelle estimée à 8,8 p. 100 (intervalle de crédibilité 95 p. $100: 5,0-16,4$ ). Cette estimation constitue une bonne indication de l'importance de la brucellose dans cette zone et confirme l'attention qui doit être accordée à cette maladie. Toutefois, il est important de prendre en considération le fait que cette estimation a été faite à partir de sérums déjà existants, dont le mode de collecte pouvait avoir une influence. Il est également important de noter que, dans l'approche bayésienne, les informations a priori utilisées ont une influence sur l'estimation de la prévalence réelle, d'où l'intérêt de considérer des opinions d'experts les plus fiables possibles. Toutefois, cette approche, en améliorant la sensibilité et la spécificité de diagnostic, et comparée à l'utilisation d'un test unique imparfait, fournit un résultat relativement fiable.

\section{Remerciements}

Les auteurs remercient M. O. N'Guessan ainsi que tous les techniciens du Laboratoire régional de Bouaké et du Laboratoire central vétérinaire de Bingerville qui ont participé à la collecte des échantillons et à la préparation de sérums. Mmes M. Marin et C. Desmedt du Cerva sont également remerciées pour leur collaboration lors des analyses sérologiques, ainsi que la Direction générale de la coopération au développement (Dgcd) pour son appui financier.

\section{BIBLIOGRAPHIE}

1. AKAKPO A.J., 1987. Brucellose animale en Afrique tropicale. Particularités épidémiologique, clinique et bactériologique. Revue Elev. Méd. vét. Pays trop., $40:$ 307-320.

2. AKAKPO A.J., BORNAREL P., 1987. Epidemiology of animal brucellosis in tropical Africa: clinical, serological and bacteriological surveys. Revue sci. tech. Off. int. Epizoot., 6: 981-1027.

3. AKAKPO A.J., BORNAREL P., D'ALMEIDA J.F., 1984. Epidémiologie de la brucellose bovine en Afrique tropicale. I. Enquête sérologique en République populaire du Bénin. Revue Elev. Méd. vét. Pays trop., 37 : 133-137.

4. ANGBA A., TRAORE A., FRITZ P., 1987. Situation de la brucellose animale en Côte d'Ivoire. Revue Elev. Méd. vét. Pays trop., 40 : 325-329.

5. BERKVENS D., SPEYBROECK N., PRAET N., ADEL A., LESAFFRE E., 2007. Estimating disease prevalence in a Bayesian framework using probabilistic constraints. Epidemiology, 17: 145-153.

6. CAMUS E., 1980. Incidence clinique de la brucellose bovine dans le nord de la Côte-d'Ivoire. Revue Elev. Méd. vét. Pays trop., 33 : 263-269.

7. CORBEL M.J., 1997. Brucellosis: an overview. Emerg. infect. Dis., 3: 213-221.

8. CORBEL M.J., 2006. Brucellosis in humans and animals. Geneva, Switzerland, WHO, $89 \mathrm{p}$.

9. Delafosse A., GOUTARD F., THEBAUD E., 2002. Epidémiologie de la tuberculose et de la brucellose des bovins en zone périurbaine d'Abéché, Tchad. Revue Elev. Méd. vét. Pays trop., 55 : 5-13.

10. DOMENECH J., 1987. Importance des brucelloses animales en Afrique centrale. Revue Elev. Méd. vét. Pays trop., 40 : 321-324. 
11. DOMENECH J., LUCET P., VALLAT B., STEWART C., BONNET J.B., BERTAUDIERE L., 1980. La brucellose bovine en Afrique centrale. II. Etude clinique et épidémiologique : particularités régionales et problèmes de l'élevage semi-intensif. Revue Elev. Méd. vét. Pays trop., 33 : 277-284.

12. FAYE B., CASTEL V., LESNOFF M., RUTABINDA D., DHALWA J., 2005. Tuberculosis and brucellosis prevalence survey on dairy cattle in Mbarara milk basin (Uganda). Prev. vet. Med., 67: 267-281.

13. GIDEL R., ALBERT J.P., MAO G.L., RETIF M., 1974. La brucellose en Afrique occidentale et son incidence sur la santé publique. Résultats de dix enquêtes épidémiologiques effectuées en Côte d'ivoire, Haute-Volta et Niger, de 1970 à 1973. Revue Elev. Méd. vét. Pays trop., 27 : 403-418.

14. JAMES A.D., 1998. Guide to epidemiological surveillance for Rinderpest. Revue sci. tech. Off. int. Epizoot., 17: 796-809.

15. MCDERMOTT J.J., ARIMI S.M., 2002. Brucellosis in sub-Saharan Africa: epidemiology, control and impact. Vet. Microbiol., 90: 111-134.

16. NIELSEN K., 2002. Diagnosis of brucellosis by serology. Vet. Microbiol., 90: 447-459.

17. PAPPAS G., PAPADIMITRIOU P., AKRITIDIS N., CHRISTOU L., TSIANOS E.V., 2006. The new global map of human brucellosis. Lancet Infect. Dis., 6: 91-99.

18. PILO-MORON E., PIERRE F., KOUAME J.B., 1979. La brucellose bovine en Côte-d'Ivoire. Epidémiologie. Revue Elev. Méd. vét. Pays trop., $32: 325-333$.

19. PRAET N., DORNY P., SAEGERMAN C., MARCOTTY T., BERKVENS D., 2006. Estimating disease prevalence and diagnostic test characteristics using a Bayesian approach. Epidémiol. Santé Anim.: 113-130.
20. PUTT S.N.H., SHAW A.P.M., WOODS A.J., TYLER L., JAMES A.D. 1988. The epidemiological approach to investigating disease problem. In: Veterinary epidemiology and economics in Africa: A manual for use in the design and appraisal of livestock health policy, 2nd Edn. Rome, Italy, FAO, http://www.fao.org/Wairdocs/ILRI/x5436E/x5436E00.htm

21. SAEGERMAN C., DE WAELE L., GILSON D., GODFROID J., THIANGE P., MICHEL P., LIMBOURG B., VO T.K.O., LIMET J., LETESSON J.J., BERKVENS D., 2004. Evaluation of three serum i-ELISA using monoclonal antibodies and protein $G$ as peroxidase conjugate for the diagnosis of bovine brucellosis. Vet. Microbiol., 100: 91-105.

22. SHEY-NJILA O., DAOUDA, NYA E., ZOLI P.A., WALRAVENS K., GODFROID J., GEERTS S., 2005. Serological survey of bovine brucellosis in Cameroon. Revue Elev. Méd. vét. Pays trop., 58: 139-143.

23. SPIEGELHALTER D.J., THOMAS A., BEST N., LUNN D., 2003. WinBUGS Version 1.4 User manuel. http://www.mrc-bsu.cam.ac.uk/ bugs/winbugs/manual14.pdf

24. THYS E., YAHAYA M.A., WALRAVENS K., BAUDOUX C., BAGAYOKO I., BERKVENS D., GEERTS S., 2005. Etude de la prévalence de la brucellose bovine en zone forestière de la Côte d'Ivoire. Revue Elev. Méd. vét. Pays trop., 58 : 205-209.

25. TOUNKARA K., MAIGA S., TRAORE A., SECK B.M., AKAKPO A.J., 1994. Epidemiology of bovine brucellosis in Mali - Serological survey and initial isolation of Brucella abortus strains. Revue sci. tech. Off. int Epizoot., 13: 777-786.

Reçu le 14.09.2007, accepté le 15.12.2008

\section{Summary}

Sanogo M., Cissé B., Ouattara M., Walravens K., Praet N., Berkvens D., Thys E. Real Prevalence of Bovine Brucellosis in the Center of Ivory Coast

During a serosurveillance survey carried out from July to August 2005 in Ivory Coast, 660 sera were collected in the administrative regions of $\mathrm{N}^{\prime} \mathrm{Zi}$ Comoe and Lacs, in the central part of the country. The sera were used to determine the real prevalence of bovine brucellosis using a Bayesian framework with four serological diagnostic tests: slow agglutination of Wright (SAW), rose bengale test (RBT), indirect ELISA (iELISA) and complement fixation test (CFT). CFT results were not taken into account in the study. The serological analyses showed individual seroprevalences of $6.2 \%$ with SAW, $5.1 \%$ with RBT and $7.0 \%$ with iELISA. The Bayesian analysis performed with the same serological results helped to estimate at $8.8 \%$ the real prevalence of brucellosis (credibility interval of 95\%: 5.0-16.4). Results show the importance of brucellosis in this part of Ivory Coast and confirm the attention that must be given to the disease.

Keywords: Cattle - Brucella - Brucellosis - Immunological technique - Morbidity - Cote d'Ivoire.

\section{Resumen}

Sanogo M., Cissé B., Ouattara M., Walravens K., Praet N., Berkvens D., Thys E. Prevalencia real de la brucelosis bovina en el centro de Costa de Marfil

Durante la encuesta de vigilancia activa de la peste bovina organizada en Costa de Marfil entre julio y agosto 2005, se recolectaron 660 sueros en las regiones administrativas de N'Zi Comoé y de los Lagos, en el centro del país. Estos sueros fueron utilizados para obtener una estimación de la prevalencia real de la brucelosis bovina, utilizando un enfoque estadístico bayesiano con cuatro tests de diagnóstico serológico: la aglutinación serológica lenta de Wright (ASW), el test de rosa bengala (TRB), el Elisa indirecto (iElisa) y el test de fijación de complemento (TFC). Los resultados de este último no fueron tomados en cuenta en este estudio. Los análisis serológicos dieron prevalencias serológicas individuales de $6,2 \%$ con la ASW, 5,1\% con TRB y 7,0\% con iElisa. El análisis bayesiano realizado con estos mismos resultados serológicos permitió obtener una estimación de la prevalencia real de la brucelosis de $8,8 \%$ (intervalo de credibilidad de 95\%: 5,0-16,4). Estos resultados constituyen una buena indicación de la importancia de la brucelosis en esta parte de Costa de Marfil y confirman la atención que se debe dar a esta enfermedad.

Palabras clave: Ganado bovino - Brucella - Brucelosis Técnica inmunológica - Morbosidad - Cote d'Ivoire. 